\begin{tabular}{cc}
\hline International Journal of Engineering \& Technology, $7(2.8)(2018) 77-81$ \\
SPC & Website: www.sciencepubco.com/index.php/IJET \\
Research Paper & International Journal of Engineering \& Technology \\
\hline
\end{tabular}

\title{
Low cost real time braille keypad for communication
}

\author{
Lakshmi Prabha. $P^{1}$ *, Sanjanamenon EJ ${ }^{1}$, Christina Samuel ${ }^{1}$, Ganga Ramesh ${ }^{1}$ \\ ${ }^{1}$ Department of Biomedical Engineering, SRM University Kattankulathur, Chennai-603203,Tamil Nadu, India \\ *Corresponding author E-mail: lakshmibmi@gmail.com
}

\begin{abstract}
Visually impaired people find it challenging to use devices such as computers, and other digital/educational software like every normal person does in this technology driven world. Due to their inability to gain access to basic and vital information they sometimes feel excluded from the society and also limit their knowledge. Several attempts have beenmade in the recent years to improve their communication methods such as tactile sign language, Braille glove, and Audioacces devices so on. This paper suggests an innovative assistive device, a Braille keypad, for the viually impaired that would help them use computers just like a normal person. The Braille keypad implements the Braille cells as 6 switches that can be pressed simultaneously based on the actual Braille combination in order to type a particular letter and words. The corresponding letters/words are displayed as well as can be heard by the user. A text to speech algorithm is implemented using puTTY software which would help the user confirm what he/she has typed is right. In this way it enables them to interact and communicate as well as use computers and mobile phones much like a normal person.
\end{abstract}

Keywords: puTTY; Braille Keypad; NVDA Speech Software; Microcontroller.

\section{Introduction}

Visual Impairment can be defined as the inability to see through naked eyes. Generally, this condition cannot be corrected by making use of spectacles or contact lenses. Vision between 20/70 and $20 / 400$ or a visual field of 20 degrees or lesser is considered as low. Visual Acuity as suggested by The Centres for Disease Control and Prevention (CDC) and World Health Organization (WHO). Blindness is defined as complete or nearly complete loss of vision. Visual impairment affects all the basic activities of people such as reading, walking and socializing with the people around. Moreover it also makes them incapable to use modern devices like Computes, Tablets, Mobilephones, etc. Studies suggest that the major causes of Visual Impairment are uncorrected refractive errors ( 43 percent), cataracts (33 percent), and glaucoma ( 2 percent). These errors can lead to myopia ,hypermetropia, astigmatism, aged related muscular degeneration, diabetic retinopathy, childhood blindness and a number of other eye related complications. Problems in the brain or trauma lead to a different type of impairment termed as Cortical Visual Impairment. When a visually impaired persons eyesight cannot be corrected to normal level it is termed as Vision impairment by the CDC. Due to the advancements in treatment and improving technology, the rates of visual impairment have reduced to a considerable amount in the present scenario.

Braille is a physical or a tactile writing system that is used by the blind or visually impaired. This system of writing or printing was invented by Louis Braille for the education of the blind. It has combinations of tangible or physical dots that can be used to represent each and every alphabet in English language as well the numbers that can be touched by the user to read and the same combinations can also be written on a specialized embossed papers with the help of a stylus. It is represented as 6 dots in a $3 \times 2$ matrix and can be readable to the visually handicapped by means of touch. Traditionally braille could be only written on embossed papers but now with the advancement in technology and innovation, Braille users are beginning to use various electronic supports too. Braille can be produced either by using a slate and stylus or can also be produced on a Braille typewriter, also called as Perkins Braille. Braille need to be produced with a Braille embosser or refreshable Braille display so as to effectively erase when any error occurs while using it otherwise it would become very tedious for the user to cross check each and every letter before writing. To convert any text to Braille document, a Braille editor or Braille translation software are required for any Braille printer. Braille can be categorized into 2 grades viz. Grade1 and Grade 2. Grade1 Figure 1 Braille or Uncontracted Braille is the one in which each letter or digit is represented as one Braille cell. Grade2 Braille or Contracted Braille is one in which group of alphabets are assigned to one or two Braille cells. Grade2 is preferred over Grade1 as it uses fewer cells and hence requires less space 10

\section{BRAILLE Alphabet}

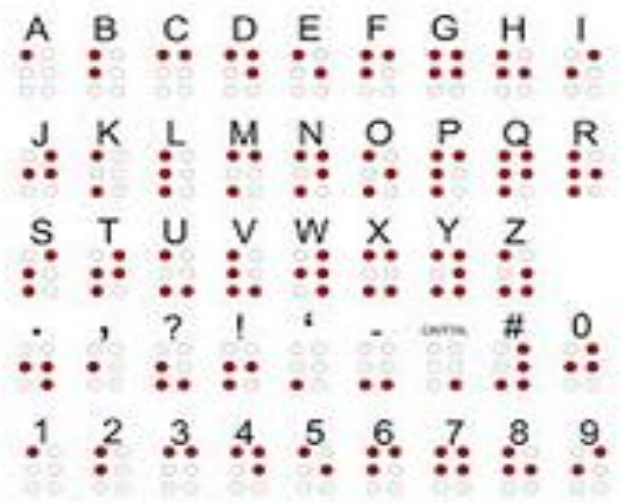

Fig. 1: Braille Alphabets and Numbers. 
In the recent years researchers have come up with various assistive technologiesand/or devices that would facilitate the visually impaired to interact and communicate as well as to educate themselves just like each and every person around. Several devices have been and are being invented that implement the Braille combination, the language of the blind and text to braille translator on chip14. A Braille glove11-12 is one such innovation which is used to send as well as receive text messages from the glove to $\mathrm{PC} /$ mobile phone and vice versa and is entirely based on the standard Braille combination. The glove comprises of flex sensors, which coincide with each finger and they are connected to a microcontroller which is programmed in accordance with the bending of the sensors. Depending on how much the corresponding sensor bends, its resistance value is transmitted to the microcontroller and the resultant value is fed to the input of servo motor According to the output of ADC values of the flex sensor, thus obtained by bending, the servo motor rotates which can be understood by the visually impaired user as the Output. A Braille to English text interface uses ATmega16 microcontroller1 wherein when a particular switch on a finger is pressed, binary 1 will be transmitted, and 0 otherwise, to the microcontroller. The Braille code, thus generated, is then converted to ASCII form by using an ASCII conversion algorithm on the microcontroller and the corresponding letters will thus be displayed on computer screen. Portable keyboard and speaker device2,7 with a braille refreshable display, for the communication between two blind or visually impaired people is also carried out. This is comparatively of lower cost and also has many possibilities for further development on the ARM-based computer system. Another model suggests 6 solenoid pins that represent the Braille cell3. An SD card is inserted and when it is detected by the device a buzzer is heard as confirmation. The user then has to select reading or writing option. The buzzer will beep on each key press also to identify if it is pressed. ATMEGA16 microcontroller will convert the braille code to English alphabet while writing and vice versa while reading by giving logic 1 to the particular actuator. A similar low cost, refreshable, portable 3 paperless braille keyboard4 is proposed for the blind which can help the visually impaired use computers based on Braille display terminal was created. ATMEGA 2506 is used in that which is programmed using $\mathrm{C}++$ language. A braille keyboard with 6 touch sensors along with 3 keys namely shift, to toggle between numbers and capital letters, back, for backspace, and space are present.The touch sensors are restricted to touch and no touch and accordingly given binary 1 and 0 which is programmed in the microcontroller and displayed on the computer screen5. Implementation of Braille to Text/Speech Converter on FPGA Spartan3 kit8,9. FPGA converts the input into corresponding English text through the decoding logic in VHDL language. After decoding, the corresponding alphabet is converted to speech through algorithm. An Electronic design of a low cost Braille Hand Glove using slot sensors and vibration motors with the help of which they can read and write emails, text messages and read ebooks13,14.Touchscreen technology has been used for braille keypad. Where novel nonvisual text input approach for touchscreen devices. With SingleTapBraille, a user enters characters including text, numbers, and punctuation by tapping anywhere on the screen with one finger or a thumb several times based on braille patterns 15 .

\section{Materials and methods}

The components used in implementing this assistive device comprises of two parts, hardware and software. The hardware consists of the tactile part wherein the Braille combination is implemented as switches along with the PIC16F877A microcontroller which is programmed to convert the Braille code to text and also to display the output. An audio enabled PuTTY software is used to display the text as well converts the text to speech format.
The hardware component encompasses of Power Supply Circuit, ButtonSwitches, Master Clear Circuit, Oscillatory Circuit, PIC16F877A Microcontroller, LCD Panel and MAX232.

a) Block Diagram

Figure 2 in block diagram has 6 keys/switches labelled 1-3 and 46 on each side respectively. The switches are placed exactly like the Braille cell combination. Depending on the combination of dots (here switches) the respective letters/numbers/characters are displayed temporarily on the LCD Panel. In addition to these 6 switches there are 3 more switches on the top right side for delete space and enter, in the same order. Delete switch, as the name implies, is used to delete/erase if any error or mistake the user makes while pressing the combination of switches. Space switch is for leaving gap between characters and enter switch is pressed to transmit and display the typed characters onto the PC via MAX232.



Fig. 2: Block Diagram of Braille Keypad.

b) PIC16F877A Microcontroller

The microcontroller controls all the operations of the keypad. It is programmed in Embedded C language using MP Lab software. The program thus fed to microcontroller is responsible for converting the braille text to readable form as well as to display it on the PC. The braille text is obtained by pressing all the switches required to display the letter simultaneously. The switches are being pressed simultaneously to avoid unnecessary confusions that would otherwise arise due to the time delay. A time delay of 5 seconds is given so as to type the next letters. The converted text is then temporarily displayed on the LCD and after pressing enter switch; the text disappears from the LCD and is displayed on the PC. On pressing the enter switch, the typed characters are transmitted via MAX232 and are displayed on the Computer screen. While displaying the typed characters the audio for each character can also be heard.

\section{c) LCD Panel}

The LCD panel temporarily displays the typed characters before displaying on the monitor. LCD panel is present as just for the purpose of confirmation. It has 2 rows and can display upto 16 characters in each row. The trimpot or trimmer potential can control the contrast of the display. The letters that are pressed using the braille keys are displayed immediately on the LCD. A time delay of 5 seconds can be seen running on the top right corner of the display. This implies that each letter has to be typed before 5 seconds elapses in order to be displayed. The correct sequence for hash needs to be pressed each time before pressing any number. The hash is temporarily displayed and then when the combination for a particular number is pressed it replaces the hash symbol on the display.

d) MAX232

It is used to transmit the converted English text from the microcontroller to the computer. The communication between the microcontroller and the computer is established due to the presence of MAX232. A serial to USB converter cable is connecter to the computer to enable the communication one end of which is connected to MAX232 IC and the other end to the computer. 


\section{e) Computer Screen}

The desired characters that are thus typed are displayed on the computer screen.puTTYsoftware is used to display the same. The software is enabled with NVDA speech software which converts the text displayed on the screen to audio format.Since visually impaired people cannot see what they have typed, this software assists them in hearing what they can typed which would help the user validate what they have typed is right. Hence, the puTTY software not only displays the typed text but also plays an audio to verify the same on the computer screen. In this way the keypad can be used by the visually impaired for communication and other interaction purposes using computer instead of a keyboard which would complicate it for them

\section{Working}

The circuit diagram depicting the working as well as various connections of the components of the model is depicted in the Figure 3 that follows. The circuit operates in $5 \mathrm{~V}$ power supply. The power supply circuit is connected to pins 11 and 32 of the microcontroller, pins 2 and 15 of the LCD display and is also connected to the 9 switches that represent the 6 Braille cell along with the special switches. The power supply circuit consists of Transformer ( $230 \mathrm{~V}$ primary and $0-9 \mathrm{~V}$ secondary), bridge rectifier, capacitors, regulator (LM7805) and resistors. A constant 5V Dc supply is supplied to each of the electronic components for their operations. There are nine button switches in total wherein six switches represent the braille cell ( 3 on each side) and the other three switches for the delete, space and enter. The rightpattern of the button switches need to be pressed simultaneously so as to the displaythe desired letters/words numbers. Numbers can also be displayed by pressing hash before pressing the combinations. Hash needs to be pressed since the combination for numbers $1-9$ and 0 are same as the combination required for the alphabets A-I and J respectively. The switches are connected to the entire B port of the microcontroller and one of the switches i.e. enter switch is connected to the $\mathrm{C}$ port of the microcontroller.

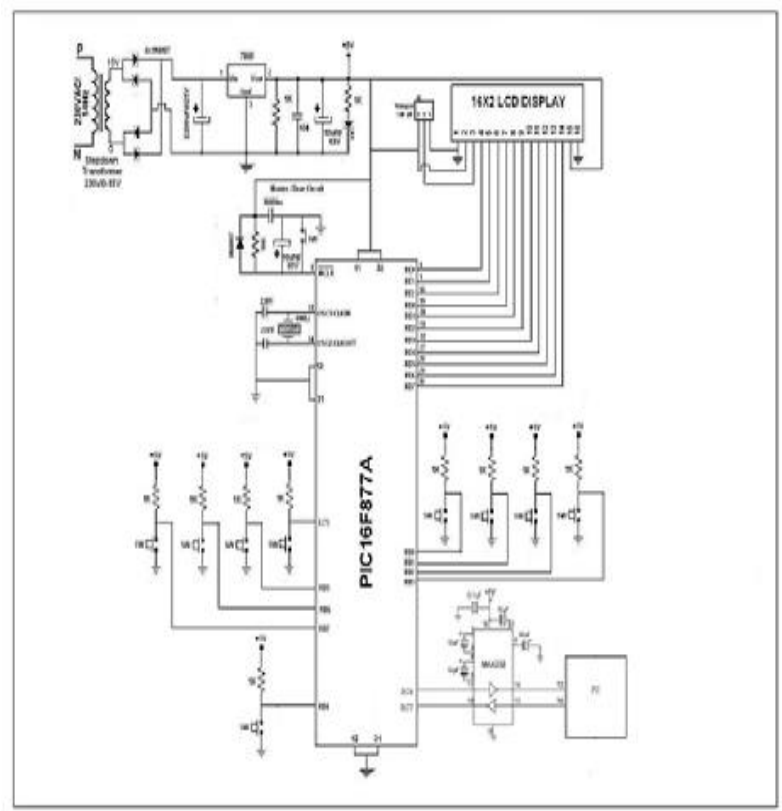

Fig. 3: Working of Braille Keypad.

In Figure 4 Final setup the pressed braille text is transferred to the microcontroller which then converts it to readable English text. The PIC16F877A microcontroller is a 40 pins IC which controls the entire operation. The inbuilt ADC makes it preferable for the application. The controller is programmed in Embedded $\mathrm{C}$ language for all the twenty six alphabets along with numbers and special characters. The synchronization and coordination of the microcontroller and its functions are controlled by the oscillator circuit. The controller is programmed in such a way that when the required combination for a particular character is pressed simultaneously the corresponding character is displayed temporarily on the LCD.Thecontroller is programmed with a 5second delay between each letter. An external master clear circuit connected to pin 1 resets the device when pressed low.

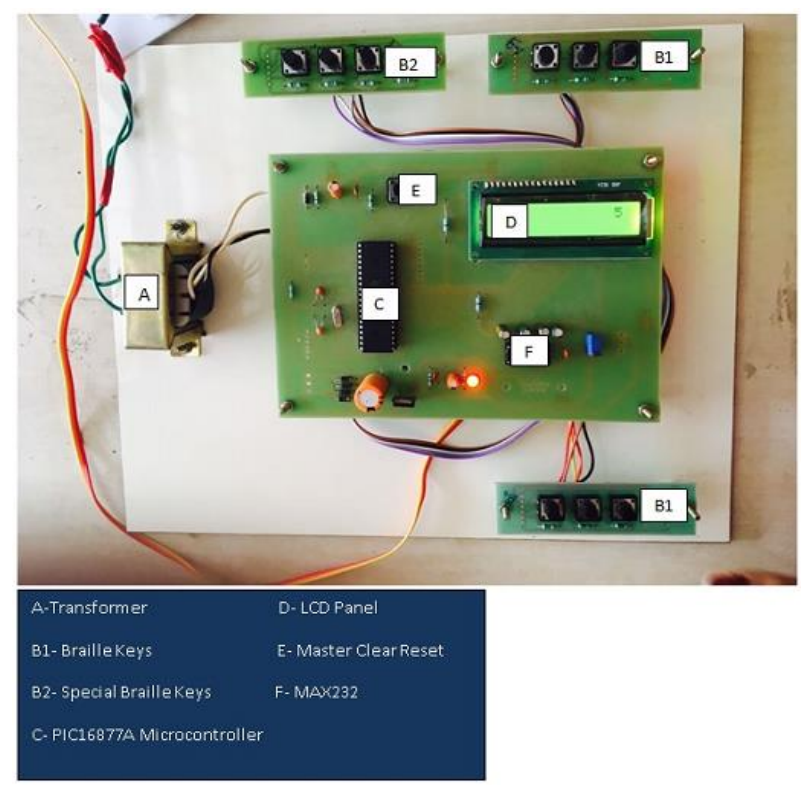

Fig. 4: Final Setup.

The $16 \times 2$ HD4478 LCD is used to display the characters immediately when the right 16pattern of buttons are pressed. The LCD can display as many as 16 characters in each of the two rows. It consists of 16 pins in total where the pin 4 to pin 11 is connected to the port RD0 to RD7 of the PIC16F877A.Pin 1and pin16 is grounded while Pin 2 and pin 15 of the LCD is connected to the power supply. Pin 3 is connected to a variable resister which works as a contrast to adjust the brightness of the display. The variable resistor or trimpot can be adjusted according to the preference of the user.The LCD can be helpful for a normal person to educate the visually impaired to educate them in using this device. The hash is displayed when it is being pressed initially and later gets replaced with the desired number that is pressed. Alphabets can be displayed both in capital and small letters. The time delay of 5 seconds can be seen running on the top right corner of the display. Once the enter switch is pressed the characters disappear from the LCD and appear on the computer screen.

MAX232 is used to transmit the converted English text in themicrocontroller to the PC. The transmission of text from the device to the computer takes place when the enter key is pressed. The cable helps transmission of the output to the computer for the display along with audio. PUTTY software is incorporated to display the characters on the PC. Once the characters are displayed on the LCD screen the user can press enter button in order to display these characters on the computer screen via MAX232. PuTTY software is network file based transfer application software which supports several network protocols and connected to serial port. The software is audio enabled with the help of NVDA speech software where the user can confirm whether the pressed character is accurate by listening to the audio.

\section{Result}

In result Figure 5 Shows words on the LCD display before displaying it on the PC. Numbers can also be displayed by pressing hash before pressing the combinations. On pressing the right sequence of switches according to the Braille combination the desired outputs can be displayed and the output can also be heard on the computer Figure 6 which would enable the user to confirm that he/she has typed the right characters. A time delay of 5 seconds is 
given so as to display the pressed The text is first displayed on the LCD and on pressing the enter key is disappears and is transmitted to the puTTY software on the PC where it gets displayed. The speech software reads out each letter and then reads the entire word for the affirmation of the user. The following pictures show the texts and numbers displayed on LCD display and puTTy software of the computer respectively. The number on the top right corner of the LCD panel is the time delay.Figure 7 and 8 are numerical example on LCD screen and PC.

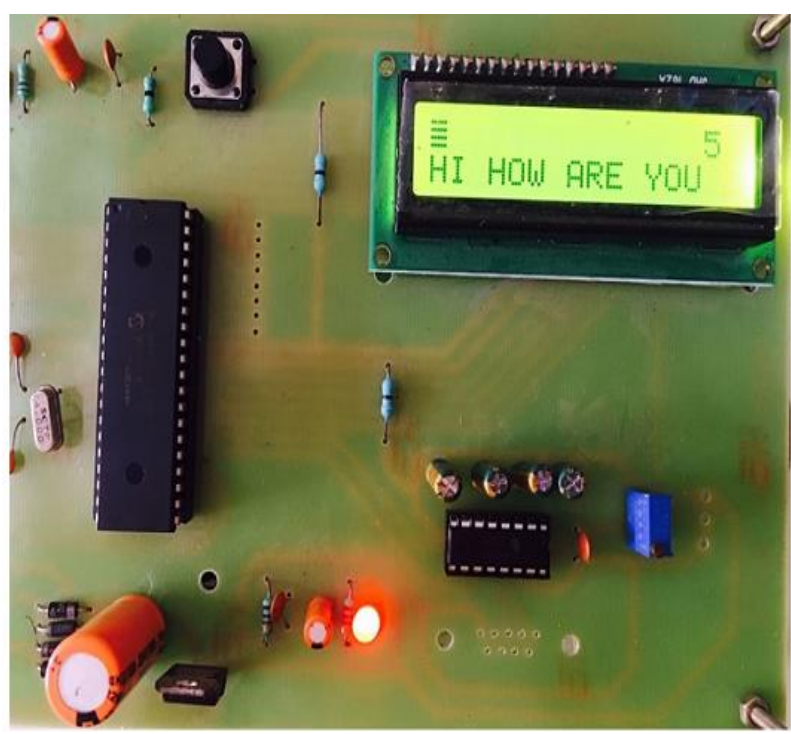

Fig. 5: Text Displayed on LCD.

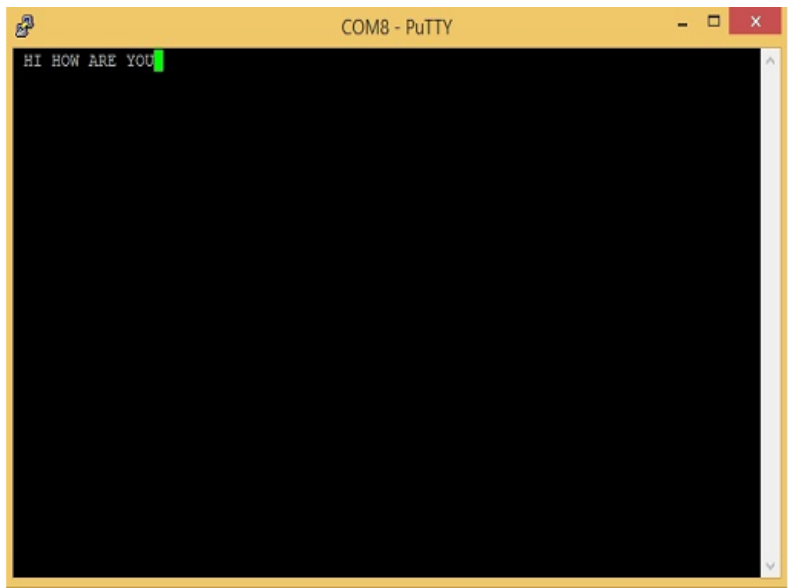

Fig. 6: Text Displayed on Computer Screen.



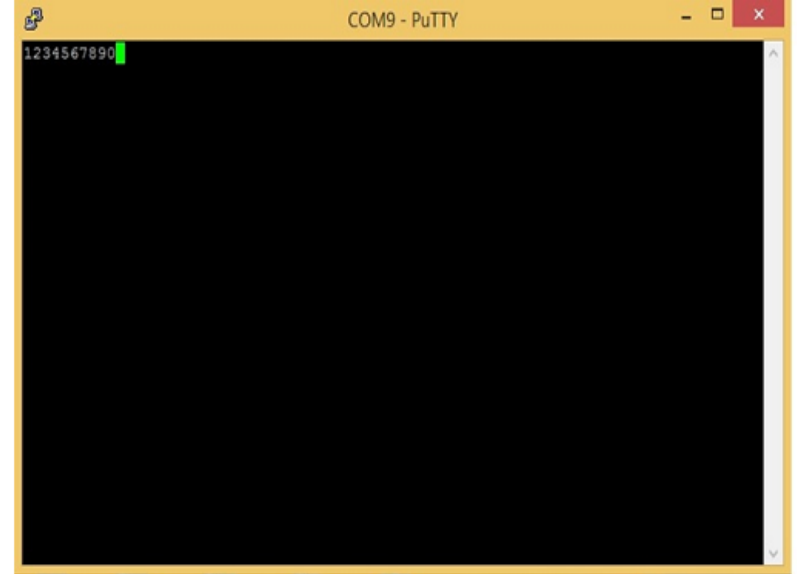

Fig. 8: Numbers Displayed on Computer Screen.

\section{Discussion}

The time delay can be reduced depending on the user because 5 secs that is given in the device presently would be longer once the user gets familiar with the operation. Special characters and and small letters are not implemented in the present model. The keypad uses only capital letters. In future the device can be programmed to toggle between the cases. The keypad can be expanded into a keyboard for the visually impaired by adding few more switches/keys such as shift key, control, alt, capslock, tab, etc. The LCD panel that is now placed can be removed and the characters can be directly displayed on the computer screen without the need to press enter key each time. Removing the LCD panel would reduce the complexity while typing. With the help of internetconnection, this device can be used for sending emails and for other means of communication and education purposes for the visually impaired

\section{Conclusion}

The objective of the project is to design a low cost portable keypadthatcanbeusedby the visually impaired instead of a keyboard while working on a computer. This keypad reduces the size and complexity that would otherwise make it difficult for the users. As all the 26 alphabets along with the 10 numbers $(0-9)$ can be representedby various sequences of the six Braille combination switches, the size of the keypadis reduced considerably and also makes to easily portable. This can also be used by the students while attempting any online competitive exams which would reduce their dependency on scribes and would also give them more confidence when attempting exams by themselves. This device would make them feel on par with normal people while using computers and other electronic devices.

\section{Acknowledgement}

We wish to thank the Department of Biomedical Engineering, SRM University, for the resources and support. A thankful response to the SRM Management, for providing excellent lab infrastructure and facilities. This study did not receive any grant from any funding agency in the public, commercial, or not-for-profit sectors. 


\section{References}

[1] Bandodkar, M. and Chourasia, V. "Low cost real-time communication braille hand-glove for visually impaired using slot sensors and vibration motors." InternationalJournal of Electrical, Computer, Energetic, Electronic and Communication Engineering, Vol. 8, 2014.

[2] Chacko, A., Devasia, J. P., and Clement, R. ."Text to braille converter.'International Journal of Emerging Technology amd Advanced Engineering, Vol. 5, 2015.

[3] C.Moore and I.Murray. "An electronic design of a low cost braille typewritter."Seventh Australian and New Zealand Intelligent information Systems Conference.10-21 november 2001

[4] Kulkarni, A. and Bhurchandi, K. "Low cost e-book reading device for blind people." International Conference on Computing Communication Control and Automation, 2015. https://doi.org/10.1109/ICCUBEA.2015.106.

[5] Kumar, Y. and Theepan, M. "An innovative braille system keyboard for the visually impaired." Electrical and Computer Engineering, 2010.

[6] Sriskanthan, N. and Subramanian, K. R.."Braille display termminal for personal computers."Nanyang Technological Institute, Singapore, Vol. 36, 1990.

[7] Sur, D., Mukherjee, B., and Gupta, V. "Braille to english text interface system with microcontroller." Advanced Research in Electrical and Electronic and Communication Engineering, Vol. 3, 2016.

[8] PrachiRajarapollu, StavanKodolikar, DhananjayLaghate, AmarsinhKhavale, "FPGA Based Braille to Text \& Speech For Blind Persons" International Journal of Scientific \& Engineering Research, Volume 4, Issue 4, April-2013

[9] Dasgupta, T.; Basu, A.; , "A speech enabled Indian language text to Braille transliteration system," Information and Communication Technologies and Development (ICTD), 2009 International Conference on , vol., no 4 issue 4 ., pp.201-211, 17-19 April 2009

[10] J. G. LmvILL, J.C Bliss, "A Direct Translation Reading Aid for the Blind," Proceedings of the IEEE, January 1966.

[11] M.Rajasenathipathi, M.Arthanari ,M.Sivakumar, "An Electronic design of a low cost Braille Hand Glove," International Journal of Advanced Computer Science and Applications, Vol. 1, No. 3, September 2010.

[12] Raj S. Mamarde, Rasika A. Lodhi, Rahul D. Bhor, "Communication system for Deaf blind people," International Journal of Engineering Research \& Technology (IJERT), ISSN: 2278-0181, Vol. 2 Issue 4, April - 2013.

[13] James D Plummer, James D Meindl, "MOS Electronics for a Portable Reading Aid for the Blind," IEEE journal of solid-state circuits, April 1972.

[14] Xuan Zhang; Ortega-Sanchez, C.; Murray, I.; "Text-to Braille Translator in a Chip," Electrical and Computer Engineering, 2006. ICECE '06. International Conference on, vol., no., pp.530-533, 1921 Dec. 2006 https://doi.org/10.1109/ICECE.2006.355685.

[15] MrimAlnfiai, SrinivasSampalli,"SingleTapBraille: Developing a text entry method based on braille patterns using a single tap", Procedia Computer Science, Volume 94, Pages 248-255,2016.

[16] T. Padmapriya and V. Saminadan, "Priority based fair resource allocation and Admission Control Technique for Multi-user Multiclass downlink Traffic in LTE-Advanced Networks", International Journal of Advanced Research, vol.5, no.1, pp.1633-1641, January 2017. https://doi.org/10.21474/IJAR01/2929.

[17] S.V.Manikanthan and K.Baskaran "Low Cost VLSI Design Implementation of Sorting Network for ACSFD in Wireless Sensor Network", CiiT International Journal of Programmable Device Circuits and Systems,Print: ISSN 0974 - 973X \& Online: ISSN 0974 9624, Issue : November 2011, PDCS112011008.

[18] A Murali, K Hari Kishore, D Venkat Reddy "Integrating FPGAs with Trigger Circuitry Core System Insertions for Observability in Debugging Process" Journal of Engineering and Applied Sciences, ISSN No: 1816-949X, Vol No.11, Issue No.12, page: 2643-2650, December 2016. 